Annals of Pure and Applied Mathematics

Vol. 21, No. 1, 2020, 69-76

ISSN: 2279-087X (P), 2279-0888(online)

Published on 21 March 2020

www.researchmathsci.org

DOI: http://dx.doi.org/10.22457/apam.v21n1a9661

Annals of

Pure and Applied

Mathematics

\title{
The Generalized Kudryshov Method Implemented to the Nonlinear Conformable Time-Fractional PHI-Four Equation
}

\author{
Md. Azizur Rahman", Md. Nur Alam and Harun-Or-Roshid \\ Department of Mathematics, Pabna University of Science and Technology \\ Pabna, 6600, Bangladesh \\ "Corresponding Author: email: azizpust@ gmail.com
}

Received 15 February 2020; accepted 20 March 2020

\begin{abstract}
This research explores the new exact solutions of the nonlinear conformable time-fractional PHI-four equation through the generalized Kudryshov method with conformable fractional derivative. The got new exact solutions are designed in styles of the rational and exponential functions designate that the studied procedure is serviceable to study the fractional nonlinear evolution equations in mathematical physics and engineering.
\end{abstract}

Keywords: Generalized Kudryshov method, nonlinear conformable time-fractional PHIfour equation, Solitary wave solutions.

AMS Mathematics Subject Classification (2010): 26A33

\section{Introduction}

Nonlinear systems of the conformable time-fractional partial differential equations (CTFPDEs) can explain mathematical models of various phenomena in distinct categories of applied physical sciences. Soliton structures to nonlinear systems of CTFPDEs are essential for investigating natural aspects in broad sectors of applied physical sciences. The performance of a symbolic computation package will obtain it functional to introduce numerous analytical techniques, for example, the extended rational sinh-cosh method [1], modified $\left(G^{\prime} / G\right)$-expansion method [2, 3], Fractional SineGordon Equation Approach [4], ( $G^{\prime} / G$ )-expansion method [5, 6, 7], New extended direct algebraic method [8], the $\exp (-\phi(\xi))$-expansion method [9, 10], the new extended direct algebraic method [11], iterative reproducing kernel Hilbert space method [13], the first integral method [14], modified khater method [15], Shifted Jacobi spectral collocation method [16], residual power series method [17], the generalized Kudryshov method [18], reproducing kernel Hilbert space method [19], and many more.

The paper applied the generalized Kudryshov method [18] to derive the different type of soliton structures for nonlinear conformable time-fractional PHI-four equation [11]. 
Md. Azizur Rahman, Md. Nur Alam and Harun-Or-Roshid

Let us consider that nonlinear conformable time-fractional PHI-four equation [11]:

$$
W_{t}^{(\mu)}-W_{x x}+a^{2} W+b W^{3}(\xi)=0, t \leq 0,0<\mu<1 .
$$

where, $a$ and $b$ are constants. The Klein-Gordon (KG) equation has worked a significant function in mathematical physics [21], as well as the KG equation, which has drawn much application in investigating solitons in condensed matter physics, in examining the interaction of solitons in a collisionless plasma and the recurrence of initial states [22]. The PHI-four equation can be studied as a particular form of the KG equation that models the phenomenon in particle physics where kink and anti-kink solitary waves interact [23]. The PHI-four equation has executed an essential role in nuclear and particle physics over the decades. Traveling wave solutions for a nonlinear variant of the Phi-4 equation are analyzed through the Weierstrass elliptic function method in [23].

\section{Fractional derivative}

We consider that $\Phi:(0, \infty) \rightarrow \mathrm{R}$, so the fractional derivative of $\Phi$ of order $\mu$ [20]:

$$
\frac{\partial^{\mu} \Phi}{\partial t^{\mu}}=\lim _{\varepsilon \rightarrow 0} \frac{\Phi\left(t+\varepsilon t^{1-\mu}\right)-\Phi(t)}{\varepsilon}, t>0, \mu \in(0,1)
$$

Some notable highlights of the fractional derivative are as follows:

$$
\begin{array}{ll}
\text { i. } & \frac{\partial^{\mu}}{\partial t^{\mu}}(a \Phi+b \Phi)=a \frac{\partial^{\mu}}{\partial t^{\mu}}(\Phi)+b \frac{\partial^{\mu}}{\partial t^{\mu}}(\Phi), \forall a, b \in R \\
\text { ii. } & \frac{\partial^{\mu}}{\partial t^{\mu}}\left(t^{\mu}\right)=\mu t^{\beta-\mu}, \forall \beta \in R \\
\text { iii. } & \frac{\partial^{\mu}}{\partial t^{\mu}}(c)=0, c=\text { const. } \\
\text { iv. } & \frac{\partial^{\mu}}{\partial t^{\mu}}(\Phi 0 \Phi)=t^{1-\mu} \Phi^{\prime}(t) \Phi^{\prime}(\Phi(t)) .
\end{array}
$$

\section{Glimpse of the generalized Kudryshov method}

Step 1: We consider that a frcational NLEE for $W(x, t)$ :

$$
K\left(\frac{\partial^{\mu} W}{\partial t^{\mu}}, \frac{\partial W}{\partial t}, \frac{\partial^{2 \mu} W}{\partial t^{2 \mu}}, \frac{\partial^{2} W}{\partial t^{2}}, \ldots \ldots \ldots .\right)=0
$$

where, $K$ represents a polynomial in $W$ and $\frac{\partial^{\alpha} W}{\partial t^{\alpha}}$ and $\frac{\partial^{2 \alpha} W}{\partial t^{2 \alpha}}$ are fractional derivatives of $W$. To locate the transformation of equation (3):

$$
W=W(x, t)=W(\xi), \quad \xi=x-\frac{c t^{\mu}}{\mu}
$$

From equation (3) and equation (4) we locate the following ODE:

$$
L\left(W, W^{\prime}, W^{\prime \prime}, W^{\prime \prime \prime}, \ldots \ldots \ldots . . .\right)=0
$$

Step 2: Calculate $M$ and $N$ through the balance rule on equation (5). 
The Generalized Kudryshov Method Implemented to the Nonlinear Conformable TimeFractional PHI-Four Equation

Step 3: Let us consider that

$$
W(\xi)=\frac{\sum_{i=0}^{N} A_{i} \psi^{i}}{\sum_{j=0}^{M} B_{j} \psi^{j}}
$$

where $A_{i}$ and $B_{j}$ are real constants, $N$ and $M$ are positive integers such that $A_{N}, B_{M} \neq 0$ and $\Psi$ satisfies the following ODE:

$$
\psi^{\prime}(\xi)=\psi^{2}(\xi)-\psi(\xi)
$$

The general solution of equation (6) is of the form:

$$
\psi(\xi)=\frac{1}{1+p e^{\xi}}
$$

where, $p$ is any arbitrary constant.

Step 4: Determine the positive integers $N$ and $M$ in equation (6) by balancing the highest order derivative term with the nonlinear term of $W(\xi)$ in equation (3) or equation (5). Moreover, we define the degree of $W(\xi)$ as $D(W(\xi))=N-M$, which gives rise to the degree of other expression as

$$
D\left(\frac{d^{q} W}{d \xi^{q}}\right)=N-M+q, D\left(W^{p}\left(\frac{d^{q} W}{d \xi^{q}}\right)^{s}=(N-M) p+s(N-M+q)\right.
$$

where, $p, q, s$ are integer numbers.

Step 5: Applying equation (6) into equation (5) and equation (9), collecting all terms with the same order of $\Phi$ together. Equating each coefficient of this polynomial to zero, yields a set of algebraic equations which can be solved to find the values of $\Phi(\xi)$ with the help of MAPLE.

\section{Solitons to the nonlinear conformable time-fractional Phi-4 equation}

Let us consider that the nonlinear conformable time-fractional Phi-4 equation:

$$
W_{t}^{\mu}-W_{x x}+a^{2} W+b W^{3}(\xi)=0, \quad t \leq 0,0<\mu<1 .
$$

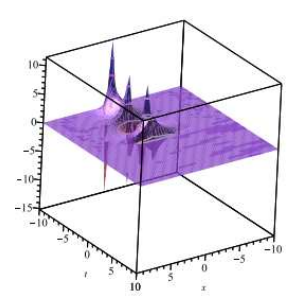

(a) Real 3D shape

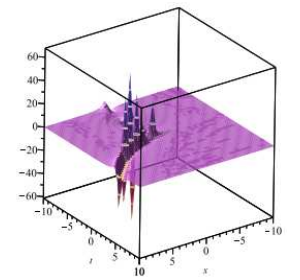

(b) Imaginary 3D shape

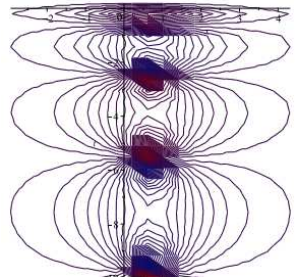

(c) Real contour plot

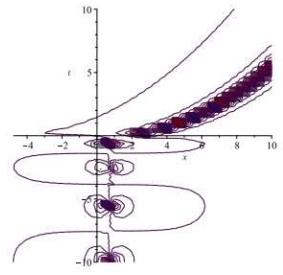

(d) Imaginary contour plot

Figure 1: The three-dimensional and contour shape of the solution in $W_{1}(x, t)$ for

$$
p=-0.5, b=1, \quad B_{0}=0.6, c=2 \text { and } \alpha=0.5 \text {. }
$$


Md. Azizur Rahman, Md. Nur Alam and Harun-Or-Roshid

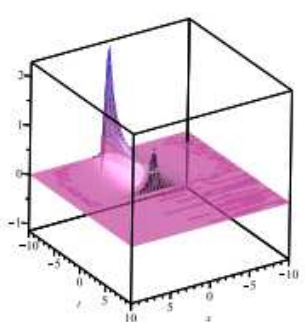

(a) Real 3D shape

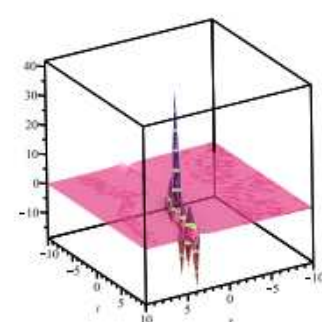

(b) Imaginary 3D shape

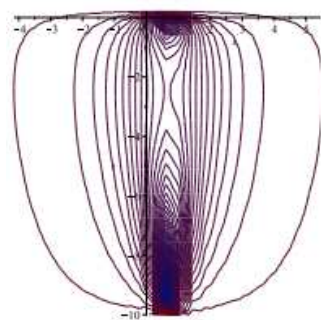

(c) Real contour plot

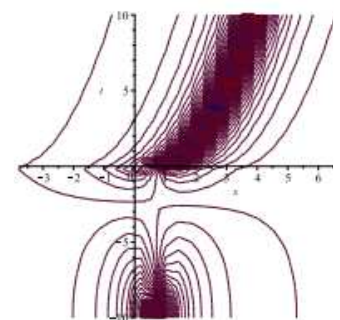

(e) Imaginary contour

(d) Plot

Figure 2: The three-dimensional and contour shape of the solution in $W_{2}(x, t)$ for

$$
p=-0.5, b=-10, \quad B_{0}=-5, c=0.5 \text { and } \alpha=0.5 \text {. }
$$

Using $W(x, t)=W(\xi)$, where $\xi=x-\frac{c t^{\mu}}{\mu}$, the equation (10) converts the following ODE:

$$
\left(c^{2}-1\right) W^{\prime \prime}(\xi)+a^{2} W+b W^{3}(\xi)=0
$$

where, $a, b$ are real parameters and $c$ is a traveling wave variable. Applying the rule of homogeneous balance on equation (11), $\left(W^{\prime \prime}(\xi)\right.$ and $W^{3}(\xi) \Rightarrow(3(N-M)=N-M+2) \Rightarrow$ $N=M+1$. Setting $M=1$ then $N=2$. Therefore, we get:

$$
W(\xi)=\frac{A_{0}+A_{1} \Psi+A_{2} \Psi^{2}}{B_{0}+B_{1} \Psi}
$$

By equation (12) and equation (11) and then equating each coefficients of $\Psi^{i}$ to zeros, we get:

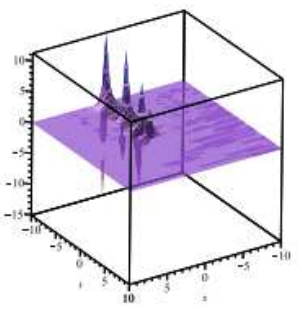

(a) Real 3D shape

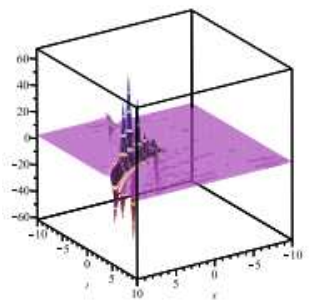

(b) Imaginary $3 \mathrm{D}$ shape
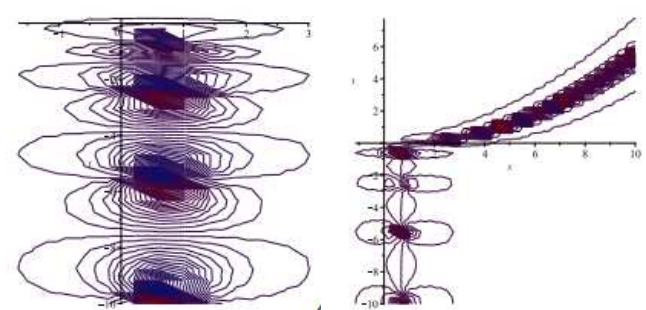

(c) Real contour plot (d) Imaginary contour Plot

Figure 3: The three-dimensional and contour shape of the solution in $W_{3}(x, t)$ for

$$
p=-0.5, b=1, \quad B_{0}=0.6, c=2 \text { and } \alpha=0.5 \text {. }
$$


The Generalized Kudryshov Method Implemented to the Nonlinear Conformable TimeFractional PHI-Four Equation

\section{The first set:}

$a=\sqrt{1-c^{2}} A_{0}=0, A_{1}=-B_{0} \sqrt{\frac{2-2 c^{2}}{b}}, A_{2}=2 B_{0} \sqrt{\frac{2-2 c^{2}}{b}}, \mathrm{~B}_{0}=B_{0}, \mathrm{~B}_{1}=-2 B_{0}$ where, $c, b$ and $B_{0}$ are constants. Using the values of the first set, equation (12) and equation (11), we have:

$$
W_{1}(\xi)=\frac{-B_{0} \sqrt{\frac{2-2 c^{2}}{b}}\left(\frac{1}{1+p e^{x-\frac{c t}{\mu}}}\right)+2 B_{0} \sqrt{\frac{2-2 c^{2}}{b}}\left(\frac{1}{B_{0}-2 B_{0}\left(\frac{1}{x-\frac{c t^{\mu}}{\mu}}\right)^{2}}\right)}{\left.1+p e^{x-\frac{c t^{\mu}}{\mu}}\right)} .
$$

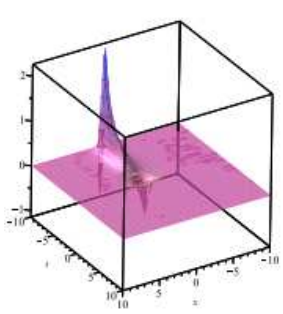

(a) Real 3D shape

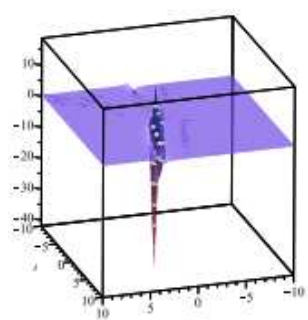

(b) Imaginary 3D shape

Figure 4: The three-dimensional and contour shape of the solution in $W_{4}(x, t)$ for

$$
p=-0.5, b=-10, \quad B_{0}=-5, c=0.5 \text { and } \alpha=0.5 \text {. }
$$

\section{The second set:}

$$
a=-\sqrt{1-c^{2}} A_{0}=0, A_{1}=B_{0} \sqrt{\frac{2-2 c^{2}}{b}}, A_{2}=-2 B_{0} \sqrt{\frac{2-2 c^{2}}{b}}, \mathrm{~B}_{0}=B_{0}, \mathrm{~B}_{1}=-2 B_{0}
$$

where, $c, b$ and $B_{0}$ are constants. Similarly, we get:

$$
W_{2}(\xi)=\frac{B_{0} \sqrt{\frac{2-2 c^{2}}{b}}\left(\frac{1}{1+p e^{x-\frac{c c^{\mu}}{\mu}}}\right)-2 B_{0} \sqrt{\frac{2-2 c^{2}}{b}}\left(\frac{1}{B_{0}-2 B_{0}\left(\frac{{ }^{-c t^{\mu}}}{\mu}\right.}\right)^{2}}{\left.1+p e^{x-\frac{c t^{\mu}}{\mu}}\right)}
$$


The third set:

$a=\sqrt{2-2 c^{2}}, A_{0}=-B_{0} \frac{2\left(c^{2}-1\right)}{b \sqrt{\frac{2-2 c^{2}}{b}}}, A_{1}=B_{0} \frac{4\left(c^{2}-1\right)}{b \sqrt{\frac{2-2 c^{2}}{b}}}, \mathrm{~A}_{2}=2 \mathrm{~B}_{0} \sqrt{\frac{2-2 c^{2}}{b}}$,

$B_{0}=B_{0}, \mathrm{~B}_{1}=-2 B_{0}$

where, $c, b$ and $B_{0}$ are constants. Similarly, we get:

$W_{3}(\xi)=\frac{-B_{0} \frac{2\left(c^{2}-1\right)}{b \sqrt{\frac{2-2 c^{2}}{b}}}+B_{0} \frac{4\left(c^{2}-1\right)}{b \sqrt{\frac{2-2 c^{2}}{b}}}\left(\frac{1}{{ }^{x-\frac{c t^{\mu}}{\mu}}}\right)+2 B_{0} \sqrt{\frac{2-2 c^{2}}{b}}\left(\frac{1}{B_{0}-2 B_{0}\left(\frac{1+\frac{c c^{\mu}}{\mu}}{1+p e^{2}}\right)}\right.}{1+p e^{x-\frac{c t^{\mu}}{\mu}}}$

The fourth set:

$a=-\sqrt{2-2 c^{2}}, A_{0}=B_{0} \frac{2\left(c^{2}-1\right)}{b \sqrt{\frac{2-2 c^{2}}{b}}}, A_{1}=-B_{0} \frac{4\left(\mathrm{c}^{2}-1\right)}{b \sqrt{\frac{2-2 c^{2}}{b}}}, \mathrm{~A}_{2}=-2 \mathrm{~B}_{0} \sqrt{\frac{2-2 c^{2}}{b}}$,

$B_{0}=B_{0}, \mathrm{~B}_{1}=-2 B_{0}$

where, $c, b$ and $B_{0}$ are constants. Similarly, we get:

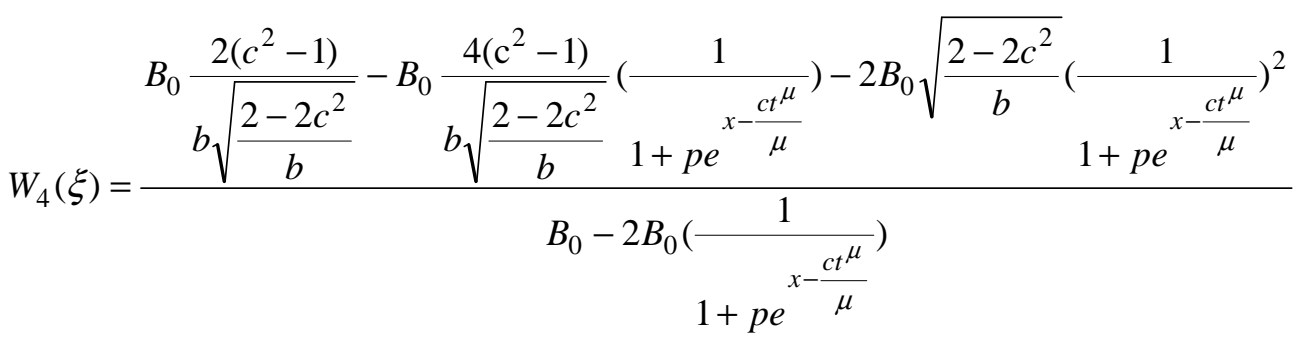

\section{Conclusions}

In this paper, we have derived diverse kinds of exact solutions which are shown in the Figures (1-4) of the equation (1) through the generalized Kudryshov method with conformable fractional derivative. The investigated way is sincere and reliable for producing new varieties of exact solutions of fractional NLEEs in mathematical physics and engineering. 
The Generalized Kudryshov Method Implemented to the Nonlinear Conformable TimeFractional PHI-Four Equation

\section{REFERENCES}

1. H.Rezazadeh, A.Korkmaz, M.M.A.Khater, M.Eslami, D.Lu and R.A.M.Attia, New exact traveling wave solutions of biological population model via the extended rational sinh-cosh method, Modern Physics Letters B, 33(28) (2019) 1950338.

2. M.N.Alam and C.Tunc, Constructions of the optical solitons and other solitons to the conformable fractional ZakharovKuznetsov equation with power law nonlinearity, Journal of Taibah University for Science, 14 (1) (2020) 94-100.

3. M.N.Alam and X.Li, New soliton solutions to the nonlinear complex fractional Schro"dinger equation and the conformable time-fractional Klein-Gordon equation with quadratic and cubic nonlinearity, Physica Scripta, DOI: https://doi.org/10.1088/1402-4896/ab6e4e, (2020).

4. H.Rezazadeh, S.M.M.Alizamini, A.Neirameh, A.Souleymanou, et al, Fractional SineGordon Equation Approach to the Coupled Higgs System Defined in TimeFractional Form, Iranian Journal of Science and Technology, Transactions A: Science, 43 (2019) 29652973..

5. M.N.Alam, M.A.Akbar and S.T.Mohyud-Din, A novel $\left(G^{\prime} / G\right)$-expansion method and its application to the Boussinesq equation, Chin. Phys. B, 23(2) 020203, (2014).

6. M.N.Alam, Exact solutions to the foam drainage equation by using the new generalized $\left(G^{\prime} / G\right)$-expansion method, Results in Physics, 5 (2015) 168-177.

7. M.N.Alam and X.Li, Exact traveling wave solutions to higher order nonlinear equations, Journal Ocean Engineering and Science, 4(3) (2019) 276-288.

8. H.Rezazadeh, K.K.Ali, M.Eslami, M.Mirzazadeh and H.Ypez-Martnez, On the soliton solutions to the space-time fractional simplified MCH equation, Journal of Interdisciplinary Mathematics, 22(2) (2019) 149-165.

9. M.N.Alam and M.M.Alam, An analytical method for solving exact solutions of a nonlinear evolution equation describing the dynamics of ionic currents along microtubules, Journal of Taibah University for Science, 11 (2017) 939-948.

10. M.N.Alam and C.Tunc, An analytical method for solving exact solutions of the nonlinear Bogoyavlenskii equation and the nonlinear diffusive predator-prey system, Alexandria Engineering Journal, 55 (2016) 1855-1865.

11. H.Rezazadeh, H.Tariq, M.Eslami, M.Mirzazadeh and Q.Zhou, New exact solutions of nonlinear conformable time-fractional Phi-4 equation, Chinese Journal of Physics, 56(6) (2018) 2805-2816.

12. M.Ilie, J.Biazar and Z.Ayati, Resonant solitons to the nonlinear Schrdinger equation with different forms of nonlinearities, Optik, 164 (2018) 201-209.

13. M.G.Sakar, A.Akgl and D.Baleanu, On solutions of fractional Riccati differential equations, Math. Advances in Difference Equations, 39 (2017).

14. O.Kolebaje, E.Bonyah and L.Mustapha, The first integral method for two fractional non-linear biological models, Discrete Continuous Dynamical Systems-S, 12(3) (2019) 487-502.

15. R.A.M.Attia, D.Lu and M.M.A.Khater, Chaos and relativistic energy-momentum of the nonlinear time fractional Duffing equation, Math. Comput. Appl. 24(1) (2019) 10.

16. E.H.Doha, M.A.Abdelkawy, A.Z.M.Amind and D.Baleanu, Shifted Jacobi spectral collocation method with convergence analysis for solving integro-differential 
Md. Azizur Rahman, Md. Nur Alam and Harun-Or-Roshid

equations and system of integro-differential equations, Nonlinear Analysis: Modelling and Control, 24(3) (2019) 332-352.

17. A.Omar, Application of residual power series method for the solution of timefractional

Schrodinger equations in one-dimensional space, Fundamenta Informaticae, 166 (2019) 87-110.

18. A.Gaber, A.F.Aljohani, A.Ebaid and J.T.Machado, The generalized Kudryashov method for nonlinear spacetime fractional partial differential equations of Burgers type, Nonlinear Dynamics, 95 (2019) 361-368.

19. A.Omar, Modulation of reproducing kernel Hilbert space method for numerical solutions of Riccati and Bernoulli equations in the Atangana-Baleanu fractional sense, Chaos, Solitons and Fractals, 125 (2019) 163-170.

20. R.Khalil, M.A.Horani, A.Yousef and M.Sababheh, A new definition of fractional derivative, Journal of Computational and Applied Mathematics, 264 (2014) 6570.

21. M.M.Khader and S.Kumar, An accurate numerical method for solving the linear fractional KleinGordon equation, Math. Methods Appl. Sci., 37(18) (2014) 29722979.

22. Ebaid, Exact solutions for the generalized Klein-Gordon equation via a transformation and Expfunction method and comparison with Adomian's method, $J$. Comput. Appl. Math., 223(1) (2009) 278-290.

23. X.Deng, M.Zhao and X.Li, Travelling wave solutions for a nonlinear variant of the PHI-four equation, Math. Comp. Model., 49(3-4) (2009) 617-622.

24. M.Ekici, Q.zhou, A.Sonmezoglu, J.Manafian and M.Mirzazadeh, The analytical study of solitons to the nonlinear Schrdinger equation with resonant nonlinearity, Optik, 130 (2017) 378-382.

25. K.Hosseini, J.Manafian, F.Samadani, M.Foroutan, M.Mirzazadeh and Q.Zhou, Resonant optical solitons with perturbation terms and fractional temporal evaluation using improved tan-expansion method and exp function approach, Optik, 158 (2018) 933-939.

26. A.Biswas, M.Ekici, A.Sonmezoglu, H.Triki, A.S.Alshomrani, Q.Zhou, S.P.Moshokoa, M.Belic, Optical solitons for Gerdjikov Ivanov model by extended trial equation scheme, Optik, 157 (2017) 1241- 1248. 